\title{
Editorial
}

\section{How has primary health care progressed? Some observations since Alma Ata}

This year (2008) it is 30 years since the Declaration of Alma Ata, USSR, 1978:

A main social target of governments, international organisations and the whole world community in the coming decades should be the attainment by all peoples of the world by the year 2000 of a level of health that will permit them to lead a socially and economically productive life. Primary health care is the key to attaining this target as part of development in the spirit of social justice.

Declaration of Alma Ata (WHO, 1978).

Fundamentally, the WHO assembly endorsed that access to health care was a basic human right and that primary health care (PHC) was the way in which this should be achieved. Since that time there has been significant global and economic change: ideologies and political beliefs have shaped health care provision, significant changes in patterns of diseases such as HIV/AIDS and SARS have altered both the shape of communities and the ways in which health planners and health practitioners respond to health needs. We are increasingly faced with new threats from terrorism, war and natural disasters such as the tsunami in SE Asia and the recent earthquake in China and cyclone in Burma, each bringing their own challenges and opportunities to health and to health care provision. Health technologies have also altered beyond recognition (there was no information highway in 1978), populations have changed towards an aging society in most countries, there is increasing global mobility and health care resources have fluctuated widely.

Central to the WHO notion of PHC was the concept of bringing health care much closer to the homes and workplaces of the people and communities who most needed health care (C) 2008 Cambridge University Press
(Bryar, 2000). It seems an appropriate time to reflect on what we have achieved in PHC and how this corresponds to the principles defined by the WHO of accessibility, use of appropriate technology, individual and community participation, increased health promotion and disease prevention and inter-sectoral co-operation and collaboration.

In 1998, the WHO consensus conference 'Primary Health Care 21: Everybody's Business' concluded that 20 years after Alma Ata (World Health Organization, 1998):

In spite of obstacles and constraints, substantial health gains have been achieved in these 20 years. Health has improved in many parts of the world and coverage with the essential elements of Primary Health Care (PHC) has expanded considerably to reach almost all populations.

Progress perhaps, but they also agreed that 'progress has been inequitable' and that in some countries or areas within countries, health has actually worsened. This is largely attributed to an increase in communicable diseases such as HIV/ AIDS, tuberculosis and malaria, and also to other problems such as violent trauma and substance abuse. Deterioration in health status is also attributed to inadequacies of PHC implementation and a lack of political commitment to allocate adequate resources to PHC. In the document it is argued that concepts such as community participation and inter-sectoral strategies 'have often not progressed beyond words'.

What evidence is there then that PHC has made any further impact on global health? Taking the PHC principle of accessibility, a rapid search of the Cochrane database of systematic reviews using the terms primary care and access revealed 
32 systematic reviews, of which three were relevant to the concept of access by people to a health care service.

The Alberta Association of Registered Nurses (2003) refer to the key component of accessibility to health services as meaning that 'all health services are universally accessible to individuals and families in the community' (2003:1).

The extent to which these three systematic reviews demonstrate this is of interest. One focuses on specialist out-reach services (Gruen et al., 2003) and concludes from nine included studies that multi-faceted out-reach clinics that work closely with primary care can improve both access and health outcomes. A second review (Briggs and Garner, 2006) focused on the integration of PHC services in low- and middleincome countries to improve delivery and health outcome. Based on three randomized cluster studies and two controlled before-after studies, the reviewers concluded that more well-designed studies are needed that should include the user side of the development as well as the service supply side. The third review was concerned with effectiveness of home-based specialist nursing services for children with acute and chronic illnesses (Cooper et al., 2006). The authors were able to find little evidence from five randomized controlled trials for any reduction in the frequency of access of hospital services.

It seems surprising that in the light of the spirit of Alma Ata there is such limited and diverse evidence to support the concept of access to health care. Whilst all three reviews call for more and better research, they are all themselves limited by the methodologies and exclusions applied. Thus, observational and qualitative studies are not included that may provide a complimentary evidence base for considering access, based on patient and practitioner experience as well as hard outcomes. Neither are analyses of policy included that would indicate the extent to which countries have improved their primary care services. Such studies would support Whitehead et al.'s (2001) contention that we should focus on 'on the ground' experiences of health if health practitioners are to gain a real understanding of what it means to be a healthy community. Of course, there are many systematic reviews and individual primary research studies that report on the effectiveness of specific interventions, we know how to treat diabetes, hypertension, cardiac failure and depression and there are protocols and guidelines based on such evidence that enable practitioners to implement these interventions. But if we do not know how to make these interventions or services universally accessible to people through PHC (especially in remote and developing countries), then their value is depleted. As part of a larger piece of work conducted on behalf of the International Council of Nurses (Kendall, 2008), I have found that there are similar issues with the other principles of PHC such as community participation and use of technology, the evidence for improvement is there but in many forms that require interpretation and careful synthesis not in ready-made systematic reviews that will help health planners to make clear decisions.

In Primary Health Care Research and Development we have published papers from all the disciplines within PHC that have shaped our knowledge base around the impact that the principles of PHC have had on health, both qualitatively as well as measurably. For example, in the last issue we published Jesper et al.'s (2008) qualitative study on the health experience of gypsy travellers in the UK. This study addresses most of the principles of PHC including access, participation, health promotion, collaboration and appropriate use of technology. In earlier issues we included school health (Brooks et al., 2007) and continence (Wagg et al., 2007). These studies provide evidence of impact and development of theory to support practice but would be unlikely, because they are largely qualitative, to be included in Cochrane style reviews. But if we want to demonstrate the true picture of progress since Alma Ata, we have to be able to develop robust methods of synthesizing and summarizing all types of evidence in primary care research and to bring this to the attention of our patients and public as well as the health planners and policy makers. We still have many lessons to learn in primary care research about the ways in which our research can influence policy and the development of the health care service; we must all take responsibility for conducting and publishing the highest quality research that will provide such evidence for the future. 


\section{References}

Alberta Association of Registered Nurses (AARN). 2003: Primary health care. Edmonton, Canada: AARN.

Briggs, C.J. and Garner, P. 2006: Strategies for integrating primary health services in middle- and low-income countries at the point of delivery. Cochrane Database of Systematic Reviews, Issue 25.

Brooks, F., Kendall, S., Bunn, F., Bindler, R. and Bruya, M. 2007: The school nurse as navigator of the school health journey: developing the theory and evidence for policy. Primary Health Care Research and Development 8, 226-34.

Bryar, R. 2000: Primary health care: does it defy definition? Primary Health Care Research and Development 1, $1-2$.

Cooper, C., Wheeler, D.M., Woolfenden, S.R., Boss, T. and Piper, S. 2006: Specialist home-based nursing services for children with acute and chronic illnesses. Cochrane Database of Systematic Reviews, Issue 4.

Gruen, R.L., Weeramanthri, T.S., Knight, S.E. and Bailie, R.S. 2003: Specialist outreach clinics in primary care and rural hospital settings. Cochrane Database of Systematic Reviews, Issue 4.

Jesper, E., Griffiths, F. and Smith, L. 2008: A qualitative study of the health experience of gypsy travellers in the UK with a focus on terminal illness. Primary Health Care Research and Development 9, 157-65.

Kendall, S. 2008: Nursing perspectives and contribution to primary health care. Geneva: International Council of Nurses, www.icn.org.ch

Wagg, A., Barron, D., Kirby, M. and Corlett, K. 2007: Development and validation of a short, easy to use questionnaire for diagnosing urinary incontinence and lower urinary tract symptoms in women: the Female Urinary Symptom Score. Primary Health Care Research and Development 8, 243-50.

Whitehead, M., Dahlgren, G. and Evans, T. 2001: equity and health sector reforms: can low-income countries escape the medical poverty trap? Lancet 358, 833-36.

World Health Organization. 1978: The declaration of Alma Ata. Geneva: WHO.

World Health Organization. 1998: Primary health care 21. Everybody's business. Geneva: WHO. 